\title{
Detection and monitoring of postinterventional success and complications of the liver using contrast-enhanced ultrasound (CEUS) - a case report after interventional treatment of a giant hemangioma
}

\author{
Giovanna Negrão de Figueiredo ${ }^{1}$, Johannes Rübenthaler ${ }^{1}$, Katharina Müller-Peltzer ${ }^{1}$, \\ Dirk-André Clevert ${ }^{1}$
}

${ }^{1}$ Department of Radiology, Ludwig-Maximilians University of Munich, Munich, Germany

\section{Introduction}

The most common benign liver lesions, with a prevalence of $3 \%$ to $20 \%$ are hemangiomas [1]. They affect females more than males and usually measure less than 5 $\mathrm{cm}$. Hemangiomas normally remain largely stable without any complications or symptoms. When a diameter of more than $5 \mathrm{~cm}$ is reached, they are referred to as giant hemangiomas (GHH) [2]. GHH are more vulnerable to complications such as abdominal pain or fullness, inflammation, fever, jaundice or even coagulations disturbances.

Regarding the imaging diagnosis, hemangiomas have specific features allowing a fast recognition by all radiological modalities, and in recent years, especially by contrast-enhanced ultrasonography (CEUS) $[3,4]$.

Received 24.04.2018 Accepted 11.06.2018

Med Ultrason

2018, Vol. 20, No 4, 536-538

Corresponding author: Dr. med. Giovanna Negrão de Figueiredo, M.D. Department of Radiology,

Interdisciplinary Ultrasound Center,

University of Munich - Grosshadern Campus

Marchioninistr. 15, 81377 Munich, Germany

Phnone: (+49) 89/44007-3627

Fax: (+49) 89/44007-8832

E-mail: Giovanna.Negrao_de_Figueiredo@ med.uni-muenchen.de
Nowadays, interventional radiologists use transcatheter arterial embolization (TAE) for hemangioma treatment [5], which is a less invasive modality compared to surgery. TAE has recently become an independent procedure for symptomatic patients with hepatic hemangiomas [6-9]. As an advantage, when compared to surgical intervention, TAE shows less periprocedural complications. The most common complications are related to necrosis or thrombosis of the hepatic hemangioma and include abdominal pain, fever and nausea. Nevertheless, other complications such as cholecystitis, biliary complications, liver abscess, liver failure or sepsis are more severe and sometimes life threatening, but infrequent to find in the literature [7].

This case report describes a postinterventional cholecystitis, a rare but important complication after TEA of GHH.

\section{Case report}

A 39-year-old woman with epigastric pain presented herself to her general practitioner, who initially performed a physical examination and a B-mode ultrasound already showing a hyperechoic lesion. Additional magnetic resonance imaging (MRI) was recommended and suggested a giant hepatic hemangioma with an extension of up to 9.5 $\mathrm{cm}$, occupying the liver segments 4,5 and 8 . 


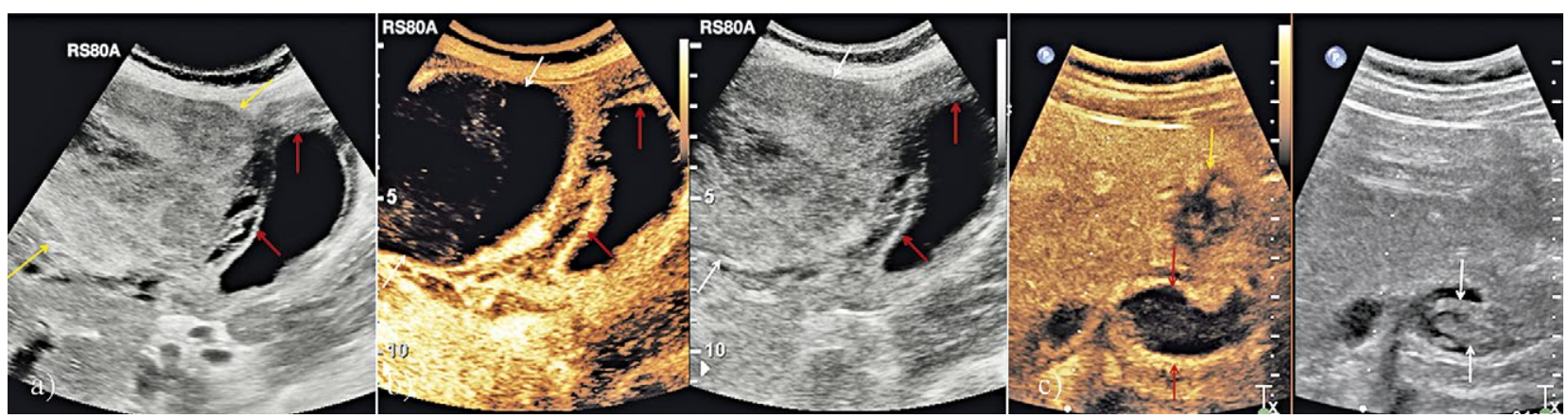

Fig 1. a) B-mode ultrasound after transcatheter arterial embolisation (TAE) of the giant hemangioma (yellow arrows) shows a gallbladder with a thickened irregularly shaped wall with multiple fluids surrounding the gallbladder (red arrows); c) CEUS (left) and native B-mode ultrasound (right) show a mostly successful devascularization of the giant hemangioma (white arrows) but a hypervascularization of the thickened gallbladder wall (red arrows) in line with sonographic features of an acute cholecystitis. The hypervascularization of the gallbladder wall is likely caused by hyperaemia due to inflammatory changes caused by the performed TAE; c) Simultaneous side-by-side ultrasound with CEUS (left) and native B-mode ultrasound (right) two weeks after initial TAE shows a thin gallbladder wall with intraluminal gallbladder-mud (white arrows) with normal perfusion of the gallbladder wall (red arrows). A residual vascularization of the giant hemangioma can also be visualized (yellow arrow).

Due to her symptoms and lesion size, the patient was referred to our radiology department for an elective TAE. Selective bland TAE of the lesion was performed using Embozene ${ }^{\circledR}$ microspheres with $400 \mathrm{~mm}$ particles. Selective catheterization images showed main vascular supply from the left hepatic artery as well as some small feeder vessels from the right and middle hepatic artery. Postembolization images were able to demonstrate a satisfactory occlusion of the vascular supply. The procedure was well tolerated without any immediate complications. Within 24 hours after intervention, the patient developed sudden onset pain in the right upper quadrant with positive Murphy's sign on physical examination. Same day ultrasound revealed an inflammation of the gallbladder with a thickened gallbladder wall $(16 \mathrm{~mm})$ and fluids surrounding the gallbladder wall (fig 1a). CEUS with a second-generation contrast agent (Sono Vue ${ }^{\circledR}$, Bracco, Italy) was recommended in order to evaluate the postembolization vascular perfusion of the GHH. The additional CEUS examination showed a hypervascularization of the gallbladder wall indicating hyperaemia due to inflammation in line with acute cholecystitis (fig 1b).

Because of the moderately elevated inflammation markers in blood tests accompanied by significant reduction of clinical symptoms, the patient was initially treated conservatively. Two days later, inflammation markers increased and the patient was treated with intravenously ciprofloxacin $400 \mathrm{mg}$ followed by oral 500 $\mathrm{mg}$ ciprofloxacin for 10 days. The patient was discharged and scheduled for elective ultrasound after two weeks, which showed a normal gallbladder wall and mud in the gallbladder. The patient was, at follow up, asymptomatic without any signs for cholecystitis. Moreover, the addi- tional CEUS showed normal perfusion of the gallbladder wall and additionally indicated a residual 50\% vascular supply of the giant hemangioma (fig 1c).

\section{Discussions}

We reported a rare case of an embolization-induced cholecystitis. Specifically, the patient presented sudden abdominal pain shortly after embolization treatment, which was successfully treated conservatively.

Cholecystitis is a rare complication of TAE of hemangiomas and if untreated, may lead to life threatening complications $[10,11]$. Although severe complications have been rarely mentioned in literature, TAE has been indiscriminately used as a non-operative treatment of hemangiomas with fewer complications than surgery [6].

Radiological imaging plays an important role not only on the target embolization but also in the detection of complications. First, angiographic anatomy is crucial for the target embolization because selective TAE relies on the confirmation of sufficient antegrade flow in the site where the microspheres will be administrated allowing admixture. Second, angiography displays the individual vascular anatomy, facilitating the right catheter position and preventing infusion of unintentional microspheres into the cystic artery [12].

The arteria cystica supplies the capillary network of the gallbladder wall and originates, in $70 \%$ of the cases, from the right hepatic artery. However, it has a very varied anatomy and may also arise from the common hepatic artery as well as from the left hepatic artery, gastroduodenal artery, superior mesenteric artery or superior pancreatico-duodenal artery among others [13]. Moreo- 
ver, the collateral perforators that supply the gallbladder body also originate from the hepatic parenchyma and the GDA.

Overall, vascular anatomy has shown that the biliary system has a blood supply coming completely from the hepatic artery while the liver cell has a double blood supply. For this reason, vascular damage of the biliary system, including the gallbladder, leads to more severe complications than vascular damage of the hepatic cell.

The embolization-induced cholecystitis, in this case, was treated conservatively. The proper treatment of a gallbladder inflammation due to TAE depends on the clinical condition of the patient in combination with imaging findings. Although CEUS findings indicated cholecystitis, clinical profile, imaging findings and laboratory values did not correlate with severity in order to require surgical intervention. The conservative treatment included supportive care such as antibiotics, pain medication and sufficient hydration and nutrition [14]. In case of persistent abdominal pain, surgical intervention is advised. However, it is normally not suggested prior to 2 weeks, as initial management should be conservative therapy.

The prognosis of this kind of cholecystitis depends on the clinical severity of the patient and the proper response to supportive care. As in this case, most patients do not require surgical intervention.

\section{Conclusion}

CEUS is a helpful tool in the detection and treatment assessment of postinterventional complications such as cholecystitis after interventional treatment of a giant hemangioma. Moreover, it is a safe technique as it does not use radiation and the contrast agent applied has no renal, thyroid or cardiac toxicity. Additionally, as a real-time imaging modality, CEUS has the possibility to directly assess the success of interventional treatment by showing the postinterventional perfusion pattern.

\section{References}

1. Semelka RC, Sofka CM. Hepatic hemangiomas. Magn Reson Imaging Clin N Am 1997;5:241-253.
2. Grieco MB, Miscall BG. Giant hemangiomas of the liver. Surg Gynecol Obstet 1978;147:783-787.

3. Sirli R, Sporea I, Popescu A, et al. Contrast enhanced ultrasound for the diagnosis of liver hemangiomas in clinical practice. Med Ultrason 2011;13:95-101.

4. Rubenthaler J, Reiser M, Clevert DA. Diagnostic vascular ultrasonography with the help of color Doppler and contrast-enhanced ultrasonography. Ultrasonography 2016;35:289-301.

5. Sun JH, Nie CH, Zhang YL, et al. Transcatheter Arterial Embolization Alone for Giant Hepatic Hemangioma. PLoS One 2015;10:e0135158.

6. Jain V, Ramachandran V, Garg R, Pal S, Gamanagatti SR, Srivastava DN. Spontaneous rupture of a giant hepatic hemangioma - sequential management with transcatheter arterial embolization and resection. Saudi J Gastroenterol 2010;16:116-119.

7. Akamatsu N, Sugawara Y, Komagome M, et al. Giant liver hemangioma resected by trisectorectomy after efficient volume reduction by transcatheter arterial embolization: a case report. J Med Case Rep 2010;4:283.

8. Seo HI, Jo HJ, Sim MS, Kim S. Right trisegmentectomy with thoracoabdominal approach after transarterial embolization for giant hepatic hemangioma. World J Gastroenterol 2009;15:3437-4339.

9. Zeng Q, Li Y, Chen Y, Ouyang Y, He X, Zhang H. Gigantic cavernous hemangioma of the liver treated by intraarterial embolization with pingyangmycin-lipiodol emulsion: a multi-center study. Cardiovasc Intervent Radiol 2004;27:481-485.

10. Sakamoto I, Aso N, Nagaoki K, et al. Complications associated with transcatheter arterial embolization for hepatic tumors. Radiographics 1998;18:605-619.

11. Karavias D, Kourea H, Sotiriadi A, Karnabatidis D, Karavias D. Gangrenous Cholecystitis Related to Transcatheter Arterial Chemoembolization (TACE) Treatment for Hepatocellular Carcinoma. J Gastrointest Surg. 2015;19:20932095.

12. Maleux G, Heye S, Vaninbroukx J, Deroose C. Angiographic considerations in patients undergoing liver-directed radioembolization with $90 \mathrm{Y}$ microspheres. Acta Gastroenterol Belg 2010;73:489-496.

13. Andall RG, Matusz P, du Plessis M, Ward R, Tubbs RS, Loukas M. The clinical anatomy of cystic artery variations: a review of over 9800 cases. Surg Radiol Anat 2016;38:529-539.

14. Forssmann K, Singer MV. Acute cholecystitis - conservative therapy. Schweiz Rundsch Med Prax 1994;83:877-879. 